\title{
Clinical and laboratory data-based nomograms for neurosyphilis diagnosis in non-HIV syphilis patients: a cross-sectional study.
}

\section{Wenjing Ge}

West China Hospital of Sichuan University

\section{Yang Zhang}

West China Hospital of Sichuan University

Chao Peng

West China Hospital of Sichuan University

Dongdong $\mathrm{Li}$

West China Hospital of Sichuan University

\section{Lijie Gao}

West China Hospital of Sichuan University

Jiajia Bao

West China Hospital of Sichuan University

Changling Li

West China Hospital of Sichuan University

Ning Chen

West China Hospital of Sichuan University

Dong Zhou

West China Hospital of Sichuan University

Li He ( $\square$ heli2003new@126.com )

West China Hospital of Sichuan University

\section{Research Article}

Keywords: Neurosyphilis, Diagnostic model, HIV-negative patient, Serum TRUST, Psychiatric symptoms

Posted Date: December 17th, 2020

DOl: https://doi.org/10.21203/rs.3.rs-123296/v1

License: (c) (i) This work is licensed under a Creative Commons Attribution 4.0 International License.

Read Full License 
Clinical and laboratory data-based nomograms for neurosyphilis diagnosis in non-HIV syphilis patients: a cross-sectional study

Wenjing Ge ${ }^{1 \dagger}$, Yang Zhang ${ }^{1 \dagger}$, Chao Peng ${ }^{1}$, Dongdong $\mathrm{Li}^{2}$, Lijie Gao ${ }^{1}$, Jiajia Bao ${ }^{1}$, Changling $\mathrm{Li}^{1}$, Ning Chen ${ }^{1}$, Dong Zhou ${ }^{1 *}$ and $\mathrm{Li} \mathrm{He}^{1 *}$

*Correspondence to: zhoudong66@yahoo.de, Dong Zhou; heli2003new@126.com, Li He

${ }^{1}$ Department of Neurology, West China Hospital, Sichuan University, Chengdu, P.R. China.

${ }^{2}$ Department of Laboratory Medicine, West China Hospital, Sichuan University, Chengdu, P.R. China.

${ }^{\dagger}$ These authors contributed equally to this work 


\begin{abstract}
Background:

The diagnosis of neurosyphilis (NS) is challenging due to the requirement of lumbar puncture and time-consuming cerebrospinal fluid tests. Therefore, a convenient high-accuracy diagnostic nomogram for NS is warranted.
\end{abstract}

\title{
Methods:
}

This cross-sectional study (108 patients) aimed to construct diagnostic models for diagnosing NS with data gathered between September 2015 and January 2020 at Western China Hospital of Sichuan University. We constructed two types of diagnostic models using 108 training samples: 1) symptoms and toluidine red unheated serum test (TRUST) (basic model) and 2) the combined model of symptoms, serum TRUST, and findings of CSF routine tests including CSF protein concentration and CSF glucose concentration (combined model). The clinical usefulness of the diagnostic models was assessed through the comparison of a receiver operating characteristic (ROC) and decision curve analysis (DCA), which adopted bootstrap resampling 500 times.

\section{Results:}

A total of 108 patients were analysed ( $82 \%$ males, mean age: 46 years). Of those, 76 were diagnosed as having reactive neurosyphilis under the criteria of positive results in both CSF treponemal and non-treponemal tests. Psychiatric symptoms and serum TRUST were the strongest diagnostic indicators in serum. A diagnostic model 
was constructed to readily provide the probability of diagnosis at point of care and presented as two nomograms. The basic model reached $79 \%$ specificity, $74 \%$ sensitivity and 0.82 Area Under the Curves (AUC) (95\% CI, 0.72-091), while the combined model showed $82 \%$ specificity, $90 \%$ sensitivity and 0.88 AUC (95\% CI, 0.80-0.94). The integrated discrimination improvement (IDI) index was 0.05 in comparison of two models.

\section{Conclusions:}

A convenient model using serum TRUST titre and presence of psychiatric symptoms was developed to indicate diagnostic results in patients suspected of NS. Two simple nomograms can be offered to clinicians to facilitate their assessment of patient diagnosis, strengthen the diagnostic decision making, enhance patient stratification, and inform patients in the clinic.

\section{Trial registration:}

This research was retrospectively registered in Ethics committee on biomedical research, West China Hospital of Sichuan University.

\section{Keywords :}

Neurosyphilis, Diagnostic model, HIV-negative patient, Serum TRUST, Psychiatric symptoms 


\section{Background}

Neurosyphilis (NS) is one of the most feared complications of syphilis [1], and the dissemination of the pathogenic bacterium of NS, Treponema pallidum (TP), to the cerebrospinal fluid (CSF) and meninges can occur at any stage of the infection [1]. Importantly, the injury of brain tissues caused by TP invasion is irreversible [1]. Trend results from syphilis notification data of the 25 countries with comprehensive surveillance systems showed an increase, especially in Europe, of up to $70 \%$ since $2000[2,3]$. However, the proportion of NS among syphilis patients is undetermined due to diagnostic limitations, requiring skilful doctors to perform lumbar puncture and lab operators for special tests [4]. Resources are usually not available to primary community healthcare centres of urban districts or common hospitals in smaller areas [5].

Prior to the advent of antibiotics, the typical symptoms of NS, such as pupil constriction when the eyes focus on a nearby object but not when the pupil is illuminated (Argyll Robertson pupils), were used to diagnose NS [1]. However, access to antibiotics has greatly increased, affecting the disease process and manifestation of NS [6]. Whether signs and symptoms can facilitate NS identification remains controversial. In recent years, headache and blurred vision are reported as supportive factors of NS diagnosis [7,8], while other reports suggest various clinical manifestations of NS lack of specificity [9]. Furthermore, most of the descriptions of 
NS symptoms come from reports on American cohorts co-infected with HIV $[8,10]$, which lack information on non-HIV NS patients, which constitute the majority of NS patients in Europe and Asia $[9,11]$.

The laboratory diagnosis of NS was putatively based on positive results from serum and CSF serologic tests, as well as elevations in CSF white-cell count and protein levels [12,13]. In 2015, an American guideline from Centers for Disease Control and Prevention (CDC), U.S. department of health and human services suggested the use of a decision tree for NS diagnosis, sequentially requiring a positive or reactive definition of non-specific, specific, or alternative tests in CSF for suspected NS patients [12]. A European guideline on European Academy of Dermatology and Venerology recommends the cut-off value of CSF treponemal tests in patients coinfected with HIV [13]. In fact, even the best threshold and combined usage of diagnostic tests for NS needs extra validation in post-antibiotics era [14].

Furthermore, combining continuous variables with clinical parameters and presenting them in a visual graph, a nomogram, which transforms a multivariable regression equation into a single numerical estimate of the probability of an event and widely used in the field of cancer diagnosis or prognosis, makes the results of a diagnostic model more simple-to-use and facilitates the evaluation of NS patients, especially in poor areas that lack expert operators and the ability to perform time-consuming tests [15]. Unfortunately, Chinese guidelines do not elaborate on the relationship between treponemal test titre and NS diagnosis, although they do recommend using CSF 
nucleated cells ( $>5$ cells/ul, positive) or CSF protein concentration $(>0.45 \mathrm{~g} / 1$, positive) as binary variables [16]. Recent studies estimating serologic cut-off values have found that the accuracy of NS diagnosis depends on the choice of controls with various clinical characteristics. In fact, both serum tests and CSF assessments have been validated as objective indicators supporting diagnosis $[8,17]$. The present study was designed to verify and explore the association between these factors and diagnostic confirmation using data from patients at the West China Hospital, Medical College of Sichuan University, from September 2015 to September 2019. Using clinical and laboratory characteristics, we developed two feasible diagnostic nomograms in order to assess the possibility of NS in an HIV-negative population with an unknown syphilis duration. 


\section{Methods}

This study included consecutive patients presenting with positive results of serum non-treponemal (Treponema Pallidum chemiluminescence assay [TP-CLIA] or Treponema Pallidum particle agglutination assay [TPPA]), and a treponemal serological test (toluidine red unheated serum test [TRUST] ) from the West China Hospital, Medical College of Sichuan University from September 2015 to September 2019. Patients met one of the following criteria: neurological or ophthalmological symptoms or signs during any stage of syphilis (such as headache, photophobia, blurred version, confusion, sleep disorders, vertigo, hearing loss, version loss, confusion, lethargy, memory change, progressive dementia, psychiatric symptoms, personality change, numbness, fatigue or pain in limbs and trunk, seizure, tremor) and no symptom) or syphilis of unknown duration, or failure of antibiotic treatment in syphilis patients (titre of serum non-treponemal test fail to decrease and fix after antibiotic treatment).

There were two types of diagnostic models: Model 1 (basic model), clinical parameters other than CSF test findings and Model 2 (combined model), combined model of both clinical parameters and CSF test findings. 


\section{Diagnostic criteria}

Subjects were enrolled at West Hospital of Sichuan University $(n=230)$. One

hundred and eight patients fulfilled all of the following criteria: (1) positive in serum

TPPA and TP-CLIA; (2) positive in both CSF-TRUST and CSF-TPPA; (3) exclusion

of HIV diagnosis (Figure 1). We applied a strict diagnostic criterion in combination of

two laboratory methods to ensure the specificity and diagnose neurosyphilis in the

suspected participants. Thus, patients with double positive results of both CSF-

TRUST and CSF-TPPA were assigned into the confirmed reactive NS group, and the others to the control group.

Figure 1. Participant flow diagram

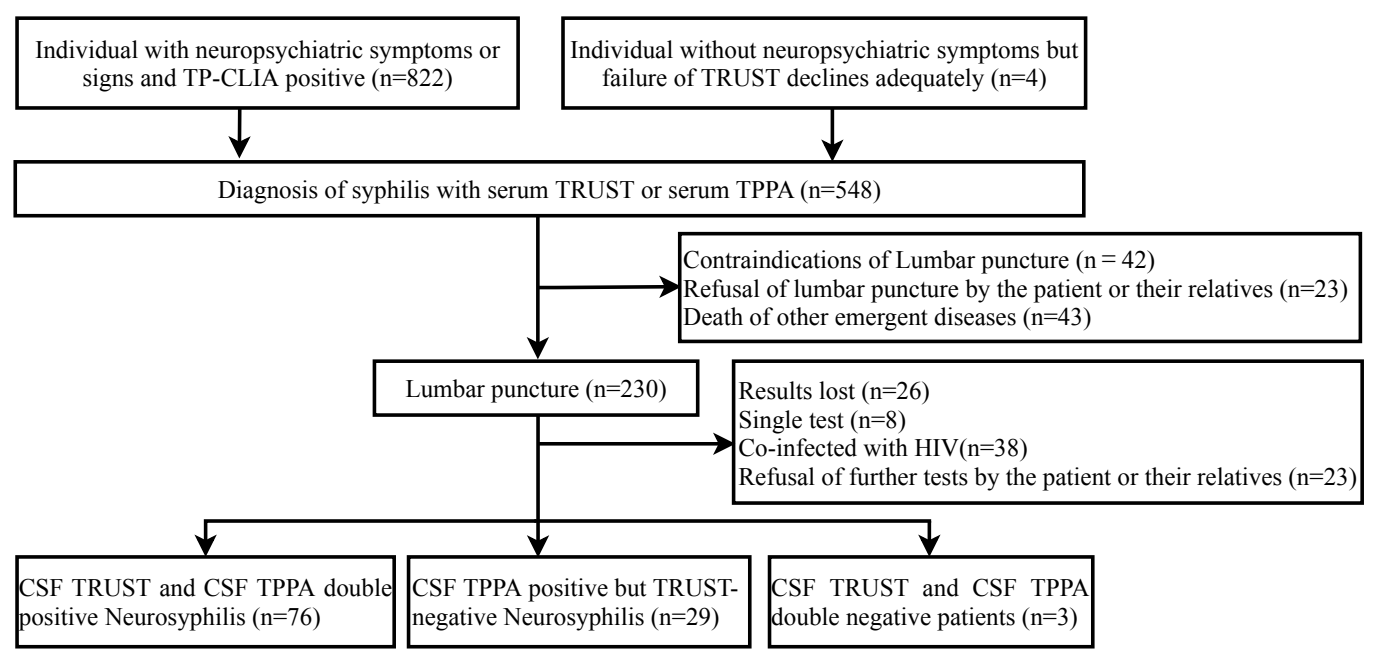




\section{Laboratory methods}

Baseline serum samples were collected within four days of the lumbar puncture [8]. Serum non-treponemal test, TRUST (Rongsheng, Shanghai, China) and treponemal test, TPPA (Fujirebio, Tokyo, Japan) or chemiluminescence assay (CLIA), and Lumipulse G TP-N syphilis (Fujirebio, Tokyo, Japan) were performed.

\section{Statistics}

Associations between categorical variables were assessed using a chi-squared test or Fisher's exact test. Associations between continuous variables and categorical variables were assessed using a Mann-Whitney U test. Diagnostic factors were analysed using univariable and multivariable regression models for confirmation of reactive NS or not-including non-reactive and not NS- as binary classification out of clinical consideration. A two-tailed $P$ value $>0.05$ was used for removal of variables. Indicators having great clinical relevance were forced back into the model. CSF items from clinical guidelines were assessed for possible additional effects. Boot strapping was resampled 500 times to obtain a $95 \%$ confidence interval and quantify the effects of diagnostic indicator selection strategies on the model development. Performance measures included the average area under the ROC curve, sensitivity, and specificity. All analyses were weighted according to the analytical guidelines. P-values $<0.05$ were considered statistically significant. R software (version 3.3.1; http://www.Rproject.org) were used for analysis. 


\section{Results}

Table 1 Baseline characteristics of the participants

\begin{tabular}{|c|c|c|c|c|}
\hline \multirow[t]{5}{*}{ Diagnosis } & Not NS & Non-reactive & Reactive NS & P- \\
\hline & \multicolumn{3}{|c|}{ NS } & \multirow[t]{4}{*}{ value* } \\
\hline & CSF TPPA- & CSF TPPA + & CSF TPPA + & \\
\hline & CSF TRUST- & CSF TRUST & CSF TRUST+ & \\
\hline & $(\mathbf{n}=3)$ & $-(n=29)$ & $(n=76)$ & \\
\hline Age, mean (sd), year & $32.3 \pm 11.0$ & $45.0 \pm 14.2$ & $47.3 \pm 11.8$ & 0.095 \\
\hline Gender (Male: Female), n & $1: 2$ & $24: 5$ & $63: 13$ & 0.151 \\
\hline Height, mean (sd), cm & $164.3 \pm 7.8$ & $165.8 \pm 7.0$ & $160.6 \pm 22.8$ & 0.899 \\
\hline Weight, mean (sd), kg & $59.0 \pm 18.2$ & $64.7 \pm 7.4$ & $62.1 \pm 12.4$ & 0.686 \\
\hline Address, n (\%) & & & & 0.034 \\
\hline City & $1(33.3 \%)$ & $16(57.1 \%)$ & $26(36.6 \%)$ & \\
\hline Urban-rural fringe area & $2(66.7 \%)$ & $1(3.6 \%)$ & $10(14.1 \%)$ & \\
\hline Village and town & $0(0.0 \%)$ & $11(39.3 \%)$ & $32(45.1 \%)$ & \\
\hline Others & $0(0.0 \%)$ & $0(0.0 \%)$ & $3(4.2 \%)$ & \\
\hline Treatment history & $0: 3$ & $21: 8$ & $55: 19$ & 0.020 \\
\hline \multicolumn{5}{|l|}{ (yes:no), $\mathrm{n}$} \\
\hline \multicolumn{5}{|l|}{ Clinical Signs or } \\
\hline Symptoms, n (\%) & & & & \\
\hline
\end{tabular}


$\begin{array}{lllll}\text { No symptom } & 0(0.0 \%) & 4(13.8 \%) & 7(9.2 \%) & 0.660\end{array}$

Psychiatric or behaviour $0(0.0 \%) \quad 9(31.0 \%) \quad 39(51.3 \%) \quad 0.051$

disorders

$\begin{array}{lllll}\text { memory change } & 0(0.0 \%) & 7(24.1 \%) & 30(39.5 \%) & 0.150\end{array}$

$\begin{array}{lllll}\text { Sleeping difficulties } & 0(0.0 \%) & 4(13.8 \%) & 21(27.6 \%) & 0.203\end{array}$

$\begin{array}{lllll}\text { Photophobia } & 0(0.0 \%) & 6(20.7 \%) & 8(10.5 \%) & 0.304\end{array}$

$\begin{array}{lllll}\text { Blurred version } & 4(13.8 \%) & 6(7.9 \%) & 0(0.0 \%) & 0.553\end{array}$

Serum tests,

$\begin{array}{lllll}\text { TP-CLIA }>100, \mathrm{n} & 2 & 29 & 76 & - \\ \text { Serum TPPA }(+:-), \mathrm{n} & 21: 0 & 68: 0 & 3: 0 & 0.068\end{array}$

Serum TRUST, Median 1:16(1:2-1:32) 1:2(1:2-1:16) 1:8(1:6-1:16) $<0.001$ (Q1-Q3)

$\begin{array}{lllll}\text { Serum creatine kinase } \quad 81.7(20.2) & 112.9(160.9) & 250.4(748.3) & 0.219\end{array}$ $1,(\mathrm{sd}), \mathrm{U} / \mathrm{L}$

Serum IgG, mean (sd), $11.3(2.2) \quad 11.8(3.6) \quad 11.9(2.6) \quad 0.689$ $\mathrm{g} / \mathrm{L}$

$\begin{array}{llll}\text { Serum albumin, mean } \quad 43.3(2.4) & 38.3(3.3) & 36.9(5.2) & 0.031\end{array}$ (sd), g/L

CSF tests, 
CSF Protein, mean (sd), $0.3(0.1) \quad 0.5(0.2) \quad 0.8(0.4) \quad<0.001$

$\mathrm{g} / \mathrm{L}$

Low level $(0.22-\quad 3(100.0 \%) \quad 17(58.6 \%) \quad 12(15.8 \%) \quad<0.001$ $0.5), \mathrm{n}$

Middle level $(0.51-\quad 0(0.0 \%) \quad 7(24.1 \%) \quad 33(43.4 \%)$

$0.79), \mathrm{n}$

High level $(0.8-2)$, n $0(0.0 \%) \quad 5(17.2 \%) \quad 31(40.8 \%)$

$\begin{array}{llll}\text { CSF Glucose, mean } \quad 3.4(0.1) & 3.7(0.6) & 3.4(0.7) & 0.011\end{array}$

(sd), g/L

$\begin{array}{lllll}\text { CSF Nucleated cell } & 3.7(5.5) & 28.9(62.2) & 37.1(86.4) & 0.081\end{array}$

(sd), cells/L

$\begin{array}{lllll}\mathrm{x}<=5, \mathrm{n} & 2(66.7 \%) & 17(60.7 \%) & 30(39.5 \%) & 0.098\end{array}$

$\mathrm{X}>5, \mathrm{n} \quad 1(33.3 \%) \quad 11(39.3 \%) \quad 46(60.5 \%)$

CSF IgG, mean (sd), $\quad 0.1(0.0) \quad 0.2(0.2) \quad 0.3(0.3) \quad<0.001$

$\mathrm{g} / \mathrm{L}$

CSF IgG Synthesis Rate $13.2(18.8) \quad 57.3(89.7) \quad 137.7(120.1) \quad<0.001$

CSF Albumin, mean $\quad 0.2(0.0) \quad 0.3(0.1) \quad 0.4(0.2) \quad<0.001$

(sd), g/L

$\begin{array}{llll}\text { CSF Generate index, } & 1.1(0.8) & 1.9(1.5) & 3.0(2.1)\end{array}$

mean (sd) 
Note: sd, standard deviation; Q, Quartile; Neurosyphilis, NS; TRUST, Toluidine red unheated serum test; TPPA, Treponema pallidum particle agglutination assay; Immunoglobulin, IgG. *Data with normal distribution was described using mean (sd).

\section{Basic characteristics}

Baseline characteristics of the 108 clinically suspected NS patients with complete information of treponemal, non-treponemal serum and CSF examination over a fouryear period are summarised in Table 1 . The median age of all study participants was 46 years old (range $17-84$ years). Men accounted for $82.9 \%$ of the 76 reactive NS patients (mean age, 47 years). The most common symptom of NS was cognitive dysfunction (31.0\%), which was mainly presented as memory change. Other symptoms included sleep disorders, photophobia, blurred version and et al. In addition, among 108 patients11(10.2\%) were non-symptomatic patients and required a CSF test. Seventy-six patients $(73.8 \%)$ did not receive any prior antibiotic treatment. Twenty-seven patients were unsuccessfully treated with nonspecific therapy for neuropsychiatric symptoms before the correct diagnosis was reached.

Table 2. Association between each indicator and diagnostic outcome

\begin{tabular}{llll}
\hline \multirow{2}{*}{ Characteristic } & \multicolumn{2}{l}{ Univariable Analysis } & \\
\cline { 2 - 4 } & Odds Ratio & $95 \%$ CI & P value \\
\hline Female & 0.74 & $0.26-2.06$ & 0.5610
\end{tabular}




\begin{tabular}{|c|c|c|c|}
\hline Age & 1.02 & $0.99-1.06$ & 0.1969 \\
\hline Treatment & 0.76 & $0.31-1.89$ & 0.5551 \\
\hline \multicolumn{4}{|l|}{ before } \\
\hline Psychiatric & 2.69 & $1.10-6.57$ & 0.0295 \\
\hline \multicolumn{4}{|l|}{ symptoms } \\
\hline No symptom & 0.71 & $0.19-2.62$ & 0.6071 \\
\hline Serum TPPA > & 2.83 & $0.96-8.38$ & 0.0599 \\
\hline \multicolumn{4}{|l|}{$1: 320$} \\
\hline \multicolumn{4}{|l|}{ Serum TRUST } \\
\hline Negative & Ref & & \\
\hline 1 & 0.00 & 0.00 -Inf & 0.9942 \\
\hline 2 & inf. & $0.00-\operatorname{Inf}$ & 0.9963 \\
\hline 4 & 1.14 & $0.17-7.60$ & 0.8901 \\
\hline 8 & 5.33 & $0.78-36.33$ & 0.0873 \\
\hline 16 & 15.11 & $2.48-92.11$ & 0.0032 \\
\hline 32 & 21.33 & $3.56-127.68$ & 0.0008 \\
\hline 64 & 18.67 & $2.55-136.41$ & 0.0039 \\
\hline 128 & inf. & $0.00-\operatorname{Inf}$ & 0.9925 \\
\hline Creatine kinase & 1.00 & $1.00-1.01$ & 0.2396 \\
\hline Serum IgG & 1.02 & $0.87-1.20$ & 0.8258 \\
\hline Serum albumin & 0.90 & $0.79-1.01$ & 0.0745 \\
\hline
\end{tabular}




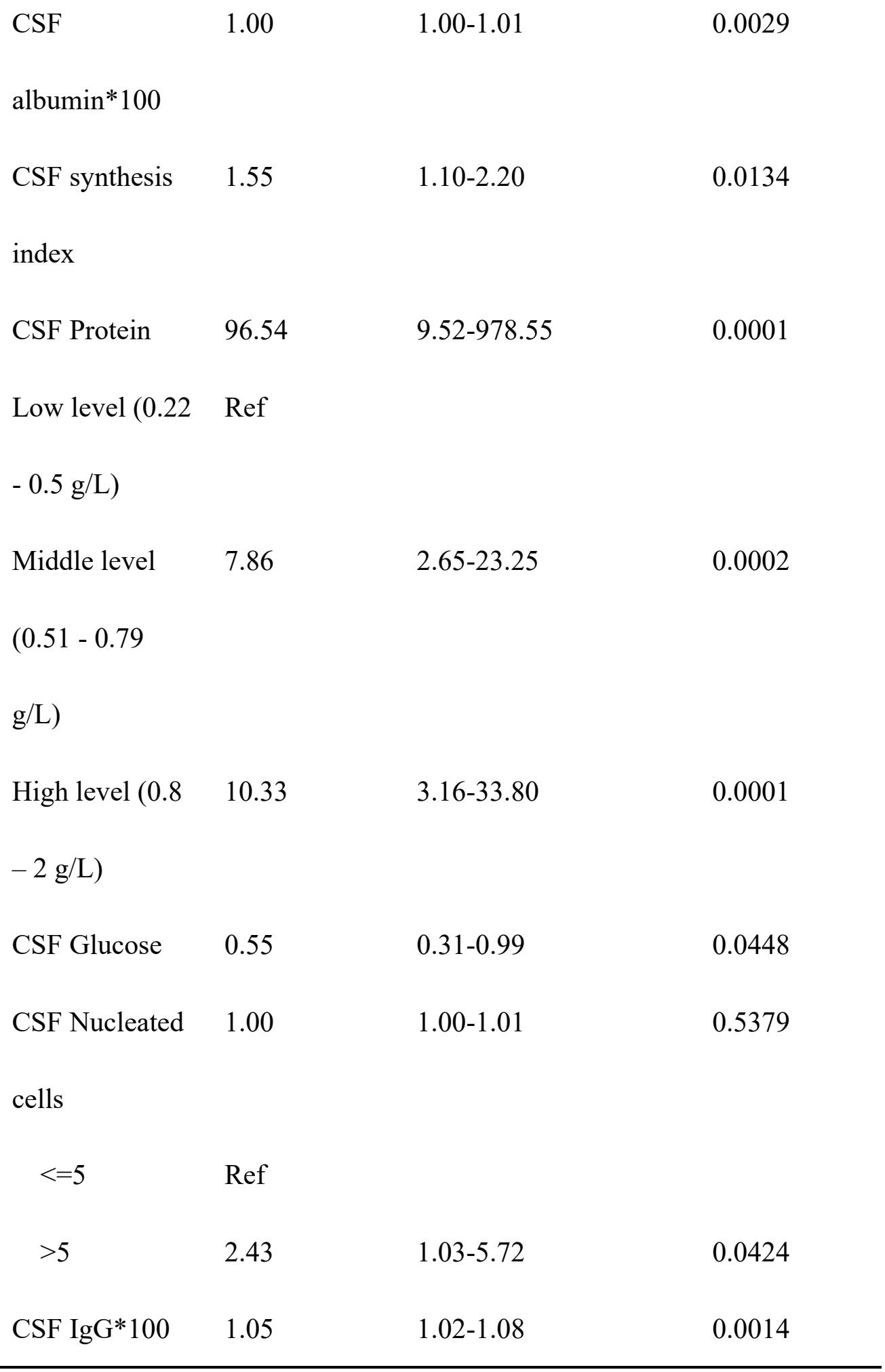

TPPA, Treponema pallidum particle agglutination assay; TRUST, Toluidine red unheated serum test; Cerebrospinal fluid, CSF; Immunoglobulin, IgG. *Data with normal distribution was described using mean (sd). 


\section{Laboratory findings and diagnostic yield}

The univariable logistic regression revealed significant differences in CSF protein levels between the reactive neurosyphilis group and the control group (odds ratio [OR], 96.54; 95\% confidence interval [CI], 9.52-978.55, P < 0.0001; Table 2). Nonspecific treponemal test (serum TRUST) findings showed incremental associations with neurosyphilis diagnosis at titre $>1: 16$ (OR, 15.11; 95\% CI, 2.48-92.11; Table 2). However, no difference in creatine kinase levels was observed between the confirmed reactive neurosyphilis and control groups (OR, 1.0; 95\% CI, 1.00-1.01). Moreover, there were no significant differences between these two groups in serum IgG and albumin levels, which contribute to the calculation of a potential effective CSF synthesis index (OR, 1.55; 95\% CI 1.10-2.20)

Table 3. Multivariable logistic analysis for the construction of diagnostic models

\begin{tabular}{|c|c|c|c|c|}
\hline & \multicolumn{3}{|c|}{ Multivariable Analysis } & \multirow{2}{*}{$\begin{array}{l}\text { Assigned } \\
\text { score }\end{array}$} \\
\hline & OR & $95 \% \mathrm{CI}$ & $\mathrm{P}$ & \\
\hline \multicolumn{5}{|l|}{ Model 1} \\
\hline Psychiatric & 4.66 & $1.33-16.28$ & 0.0160 & 5 \\
\hline Serum TRUST & & & & \\
\hline
\end{tabular}




\begin{tabular}{|c|c|c|c|c|}
\hline 0 & 1.0 & & & \\
\hline 8 & 7.61 & $1.27-45.73$ & 0.0266 & 8 \\
\hline 16 & 22.57 & $4.28-119.10$ & 0.0002 & 23 \\
\hline 32 & 23.12 & $4.72-113.10$ & 0.0001 & 23 \\
\hline 64 & 32.16 & $5.16-200.43$ & 0.0002 & 32 \\
\hline \multicolumn{5}{|l|}{ Model 2} \\
\hline Psychiatric & 4.74 & $1.09-20.56$ & 0.0375 & 5 \\
\hline \multicolumn{5}{|c|}{ Serum TRUST } \\
\hline 0 & 1.0 & & & \\
\hline 8 & 9.63 & $1.37-67.51$ & 0.0226 & 10 \\
\hline 16 & 21.55 & $3.46-134.36$ & 0.0010 & 22 \\
\hline 32 & 17.17 & $2.98-98.86$ & 0.0015 & 17 \\
\hline 64 & 71.42 & $7.22-706.72$ & 0.0003 & 71 \\
\hline \multicolumn{5}{|l|}{ CSF protein } \\
\hline 0 & 1.0 & & & \\
\hline$>5.1$ & 5.28 & $1.33-21.00$ & 0.0183 & 5 \\
\hline CSF glucose & 0.31 & $0.12-0.78$ & 0.0137 & - \\
\hline
\end{tabular}

TRUST, Toluidine red unheated serum test; Immunoglobulin, IgG; Cerebrospinal fluid, CSF 
Multivariable logistic analyses (model 1) indicted that the following factors were more likely related to confirmed NS: serum TRUST 1:8 (OR, 7.61; 95\% CI, 1.27 45.73), 1:16 (OR, 22.57; 95\% CI, 4.28-119.10), 1:32 (OR, 23.12; 95\% CI, 4.72113.10), and 1:64 (OR, 32.16; 95\% CI, 5.16-200.43) and psychiatric symptoms (OR, 4.66; 95\% CI, 1.33-16.28) (Table 3). Based on model 2, when CSF protein $>5.1(\mathrm{OR}$, 5.28; 95\% CI, 1.33-21.00) and CSF glucose (OR, 0.31; 95\% CI, 0.12-0.78) were added into the calculation, serum TRUST and psychiatric symptoms remained significantly associated with confirmed NS $(\mathrm{P}<0.05)$.

Table 4. ROC Curves, AUC and nomograms of models for NS diagnosis using multivariable logistic regression modelling

\begin{tabular}{|c|c|c|c|c|c|c|c|c|c|}
\hline \multirow{3}{*}{ Test } & ROC area & Best & Specifici & Sensitiv & Accura & Diagno & Positiv & Negative & \multirow{3}{*}{$\mathrm{P}$} \\
\hline & (AUC) & thres & ty & ity & cy & se-OR & e-pv & \multirow[t]{2}{*}{$-p v$} & \\
\hline & $95 \% \mathrm{CI}$ & hold & & & & & & & \\
\hline Model & 0.8236 & 1.093 & 0.7931 & 0.7361 & 0.7525 & 10.693 & 0.8983 & 0.5476 & 0.0542 \\
\hline \multirow[t]{2}{*}{1} & $(0.72-$ & 3 & & & & & & & \\
\hline & \multicolumn{3}{|l|}{ 0.909) } & & & & & & \\
\hline Model & 0.8764 & 0.955 & 0.8966 & 0.8194 & 0.8416 & 39.333 & 0.9516 & 0.6667 & \\
\hline \multirow[t]{2}{*}{2} & $(0.55-$ & 7 & & & & 3 & & & \\
\hline & 0.9369) & & & & & & & & \\
\hline
\end{tabular}


Receiver operating characteristics (ROC) curve of model 1 (basic model) was generated using psychiatric symptoms and serum TRUST, and that of model 2 (combined model) was generated using CSF protein and CSF glucose for predicting neurosyphilis. Diagnose-odds ratio (OR), positive predictive value (pv), and negative pv were calculated. *Area Under Curve (AUC) confidence interval and significance test adopted 500-time bootstrap resampling

The sensitivity of model $1(73.61 \%)$ was lower than model $2(81.94 \%)$, as was the specificity of model $1(79.31 \%)$ versus model $2(89.66 \%)$. The accuracies of model 1 and model 2 were 0.7525 and 0.8614 , respectively (table 4 ). The positive predictive values of model 1 and model 2 were 0.8983 and 0.9516 , while their negative predictive values were 0.5476 and 0.6667 , respectively. 
Figure 2. ROC curve, DCA and nomograms
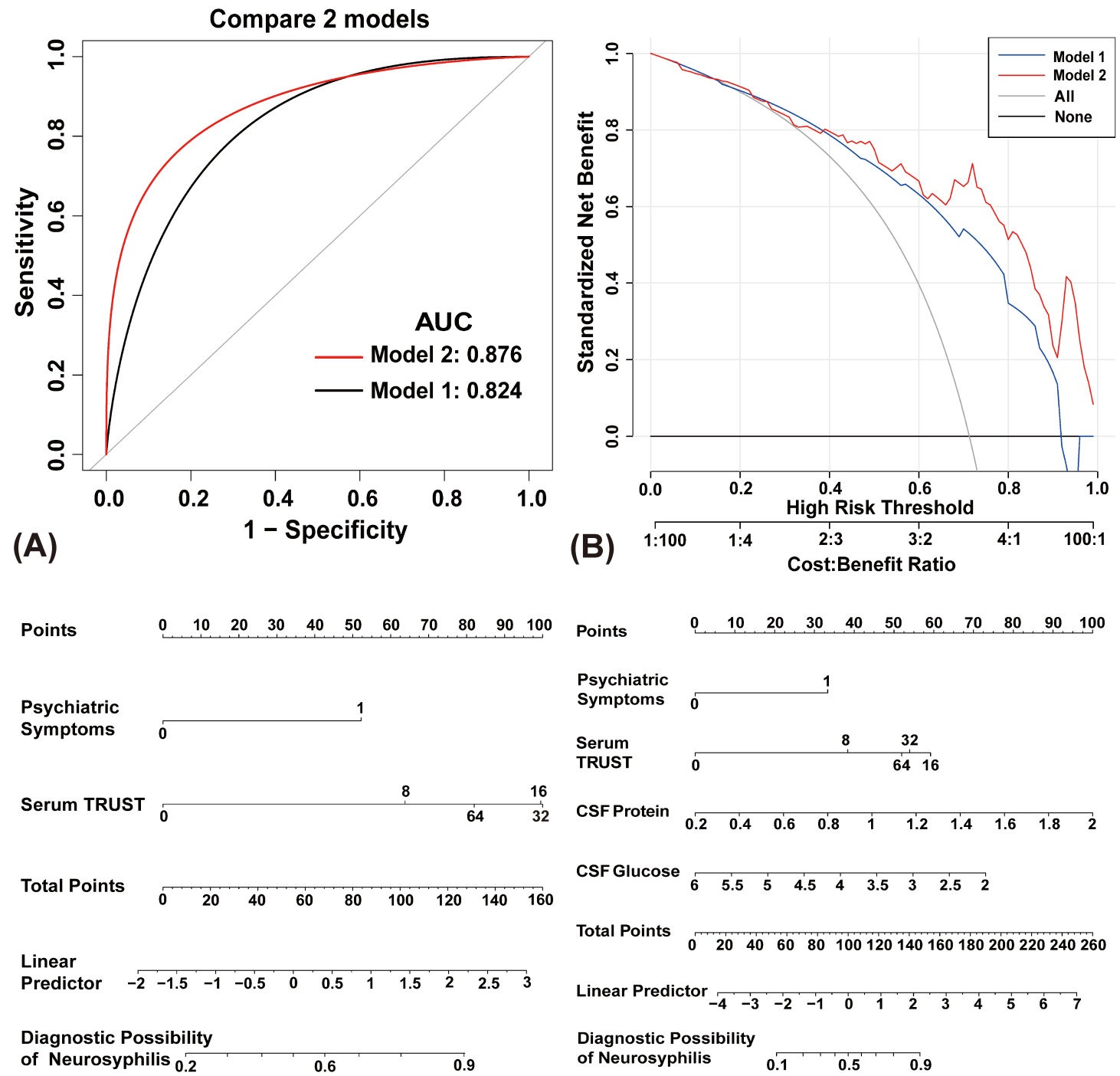

(C)

(D)

(A) AUC of Model 1 (Basic model) and Model 2 (Combined model). P value $=0.02$ in comparison between Model 1 and Model 2. (B) DCA of Model 1 and 2 (C) A nomogram visualizing the multi-variable diagnostic model of Model 1. (D)

Nomogram of Model 2. *AUC confidence interval and significance test adopt Bootstrap resampling 500 times 
Both models were useful between ROC 72\%-93\%. Figure 2 illustrates the decision curves for Models 1 and 2 for the diagnostic probability of NS. The integrated discrimination improvement (IDI) index was $0.19(95 \% \mathrm{CI}, 0.00-0.37)(\mathrm{p}=0.04$, standard error, 0.02). 


\section{Discussion}

In this study, two important clinical findings were achieved. First, serum TRUST was a useful indicator for the diagnosis of NS. Second, diagnostic models combining clinical parameters and CSF protein had clinical value. A diagnostic model based on nanogram of NS using clinical characteristic and laboratory tests was built as well.

High serum TRUST is common in syphilis patients, but in this study, serum TRUST differentiated reactive NS patients from suspected NS patients. As compared with non-reactive NS and precluded patients, the levels of serum TRUST were higher in reactive NS. Cai et al. also indicated a 5-fold increased likelihood of asymptomatic NS in patients with a serum TRUST titre $\geq 1: 64$ [17]. Researchers noted that an increase in serum TPPA titre and serum creatine kinase could serve as a surrogate for CSF clinical abnormalities after lumbar punctures $[17,18]$. Unfortunately, we were unable to determine titre grades of serum TPPA, since the laboratory system of our hospital automatically sets and reports serum TPPA $>1: 320$ as positive. Nonetheless, once infected, serum TPPA remains positive during a patient's lifetime, so we did not include this item in order to minimize the false positive rate. Xiao et al. indicated elevated serum creatine kinase may indicate neurosyphilis among HIV-negative syphilis patients [18]. However, our data showed no significant difference in serum creatine kinase among reactive NS, non-reactive NS and not NS. 
We then constructed diagnostic models for NS, combing clinical parameters and serum TRUST. When combined with clinical parameters, the diagnostic performance improved compared to the use of serum TRUST alone. NS was relatively common among males in our cohort, which is similar to British reports from Public Health England [19]. However, the rate of NS diagnosis was not significantly different. Instead, the significant clinical parameters were psychiatric symptoms and memory deterioration.

A DCA was executed to assess diagnostic model performance. The net benefit of Model 2 was better than that of the other model with threshold probabilities of 40$80 \%$. When we consider that lumbar puncture may be difficult for patients, using Model 1 and having about $80 \%$ diagnostic probability may be sufficient to diagnose a patient with NS. However, in most cases, an $80 \%$ threshold probability is not sufficient, especially in cases with a high threshold to perform continuous intravenous antibacterial therapy. In such cases, it is better to complete CSF tests and conduct more specific diagnostic tests, such as Venereal Disease Research Laboratory (VDRL) in CSF, a well-known specific test, broadly used to diagnose idiopathic NS in America but time-consuming and with low feasibility, especially in countries with high rates of sick patients [20]. The results of this study offer a sensitive screening nomogram for choosing candidates to undergo lumbar puncture, complete CSF regular tests or measure CSF-VDRL. 
This study had several limitations. First, the study might suffer from sampling bias. We did not exclude patients who received insufficient antibiotic therapy before lumbar puncture. The number of Treponema pallidum correlated with disease activity in patients, but we did not exclude such cases. Among those, the diagnosis was set as a binary variable. Under this criterion of group assignment, false negatives were possible due to non-reactive NS cases. In theory, disease duration should have been analysed as a risk factor for NS, but it is difficult for patients with neuropsychological symptoms to provide the exact time of syphilis infection or information on sexual activities. Additionally, another limitation was the small sample size and that the sophisticated pathological categories of NS were not employed here. Whether patients in each dedicated category had a different prognosis or not remains unknown due to lack of follow-up investigation. We are building up a systematic database and prospectively designing new studies to improve the quality of the evidence and facilitate more comprehensive patient care. Lastly, in order to verify the validity of this model, future studies are warranted.

\section{Conclusions}

The present study showed that psychiatric symptoms, serum TRUST, and CSF protein correlated with a diagnosis of NS in non-HIV NS. Further, a convenient score model was developed to indicate diagnostic results in suspected NS patients with or without a lumbar puncture. Importantly, two nomograms can be offered to clinicians 
to improve their abilities to assess patient diagnosis, strengthen diagnostic decision making and inform patients in the clinic. To increase its applicability, future studies should focus on internal improvement and external validation.

\section{List of abbreviations:}

CSF: cerebrospinal fluid

NS: neurosyphilis

TP: Treponema pallidum

TPCA: Treponema pallidum chemiluminescence assay

TPPA: Treponema pallidum particle agglutination assay

TRUST: toluidine red unheated serum test

VDRL: Venereal Disease Research Laboratory

\section{Declarations:}

\section{Ethics approval and consent to participate}

All methods were carried out in accordance with Transparent Reporting of a multivariable prediction model for Individual Prognosis or Diagnosis (TRIPOD) guidelines and regulations. 
Ethics committee on biomedical research, West China Hospital of Sichuan University approved the study and waived informed consent from all subjects. The committee's reference number was 1163 in 2020 approval.

\section{Consent for publication}

Not applicable

\section{Availability of data and materials}

The datasets used and/or analysed during the current study available from the corresponding author on reasonable request.

\section{Competing interests}

The authors declare that they have no competing interests

\section{Funding}

This work was funded by the National Key Research and Development Program of China (Nos. 2018YFC1311400, 2018YFC1311401) and Clinical Research Incubation Project of West China Hospital of Sichuan University (Grant 2018HXFH022)

\section{Authors' contributions}

Wenjing Ge analysed and interpreted the patient data regarding neurosyphilis and was a major contributor in writing the manuscript. Yang Zhang and Li He instructed the study design of clinical research. Chao Peng performed part of lumber puncture. Dongdong Li instructed the interpretation of laboratory tests. Lijie Gao instructed the data processing. Jiajia Bao and Changling Li helped process ethics profile. Chen Ning 
and Li He provided the support of project grants. Dong Zhou proposed the study

direction of neurosyphilis. All authors read and approved the final manuscript.

\section{Acknowledgements}

Not applicable

\section{References}

1. Ropper AH. Neurosyphilis. N Engl J Med. 2019 Oct 3;381(14):1358-1363. doi: 10.1056/NEJMra1906228. Erratum in: N Engl J Med. 2019 Oct 31;381(18):1789. PMID: 31577877.

2. Dombrowski JC, Pedersen R, Marra CM, Kerani RP, Golden MR. Prevalence Estimates of Complicated Syphilis. Sex Transm Dis. 2015 Dec;42(12):702-4. doi: 10.1097/OLQ.

3. CDC Sexually Transmitted Diseases Surveillance. 2015. http://www.cdc.gov/std/stats15/sysphilis.http

4. Tang W, Huang S, Chen L, Yang L, Tucker JD, et al. Late Neurosyphilis and Tertiary Syphilis in Guangdong Province, China: Results from a Crosssectional Study. Rep, 2017, 7:45339.

5. Zhu L, Gu X, Peng RR, Wang C, Gao Z, et al. Comparison of the cerebrospinal fluid (CSF) toluidine red unheated serum test and the CSF rapid plasma reagin test with the CSF venereal disease research laboratory test for diagnosis of neurosyphilis among HIV-negative syphilis patients in China. J Clin Microbiol. 2014 Mar;52(3):736-40. doi: 10.1128/JCM.02522-13.

5. Clement ME, Okeke NL, Hicks CB. Treatment of syphilis: a systematic review. JAMA. 2014 Nov 12;312(18):1905-17. doi:

10.1001/jama.2014.13259.

6. Dumaresq J, Langevin S, Gagnon S, Serhir B, Deligne B, et al. Clinical prediction and diagnosis of neurosyphilis in HIV-infected patients with early Syphilis. Journal of Clinical Microbiology, 2013, 51(12):4060-4066.

7. Marra CM, Maxwell CL, Smith SL, Lukehart SA and Barnett SH. Cerebrospinal Fluid Abnormalities in Patients with Syphilis: Association with Clinical and Laboratory Features. The Journal of Infectious Diseases, 2004, 189(3):369-376.

8. Zhang HL, Lin LR, Liu GL, Zeng YL, Wu JY, et al. Clinical Spectrum of Neurosyphilis among HIV-Negative Patients in the Modern Era[J]. Dermatology, 2013, 226(2):148-156.

9. Davis AP, Stern J, Tantalo L, Sahi S, Holte S, et al. How Well Do Neurologic Symptoms Identify Individuals With Neurosyphilis? Clin Infect Dis. 2018 Jan 
18;66(3):363-367. doi: 10.1093/cid/cix799

10. Choe PG, Song JS, Song KH, Jeon JH, Park WB, et al. Usefulness of routine lumbar puncture in non-HIV patients with latent syphilis of unknown duration[J]. Sexually Transmitted Infections, 2010.

11. Workowski KA, Bolan GA. Centers for Disease Control and Prevention. Sexually transmitted diseases treatment guidelines, 2015. MMWR Recomm Rep. 2015 Jun 5;64(RR-03):1-137.Janier M, Hegyi V, Dupin N, Unemo M, Tiplica GS, et al. 2014 european guideline on the management of syphilis. Journal of the European Academy of Dermatology \& Venereology, 2014 28(12), 1581-93.

12. Tuddenham S, Ghanem KG. Neurosyphilis: Knowledge Gaps and Controversies. Sex Transm Dis, 2018 March; 45(3):147-151.

13. Kingston M, French P, Higgins S, McQuillan O, Sukthankar A, et al. Members of the Syphilis guidelines revision group 2015, Radcliffe K, Cousins D, FitzGerald M, Fisher M, Grover D, Higgins S, Kingston M, Rayment M, Sullivan A. UK national guidelines on the management of syphilis 2015. Int J STD AIDS. 2016 May;27(6):421-46. doi: 10.1177/0956462415624059

14. Chinese Sexual Transmitted Disease Center. Guidelines for diagnosis and treatment of syphilis, gonorrhea, genital herpes, genital tract Chlamydia trachomatis infection,Chinese Journal of Dermatology, 2014, 47(5). doi: 10.3760/cma.j.issn.0412-4030.2014.05.022

15. Dumaresq J, Langevin S, Gagnon S, Tremblay C, et al. 2013. Clinical prediction and diagnosis of neurosyphilis in hiv-infected patients with early syphilis. Journal of Clinical Microbiology, 51(12), 4060-4066.

16. Xiao Y, Tong ML, Liu LL, Lin, LR, Chen MJ, et al. Novel predictors of neurosyphilis among HIV-negative syphilis patients with neurological symptoms: an observational study[J]. BMC Infectious Diseases, 2017, 17(1):310.

17. Public Health England. Addressing the increase in syphilis in England: Phe action plan, 2019.

Available: https://assets.publishing.service.gov.uk/government/uploads/system /uploads/attachment_data/file/806076/Addressing_the_increase_in_syphilis_i n_England_Action_Plan_June_2019.pdf. Accessed 24 Jun 2019.

18. Marra CM, Tantalo LC, Maxwell CL, Ho EL, Sahi SK, Jones T. The rapid plasma reagin test cannot replace the venereal disease research laboratory test for neurosyphilis diagnosis. Sex Transm Dis. 2012;39(6):453-457. doi: 10.1097/OLQ.0b013e31824b1cde 
Figures

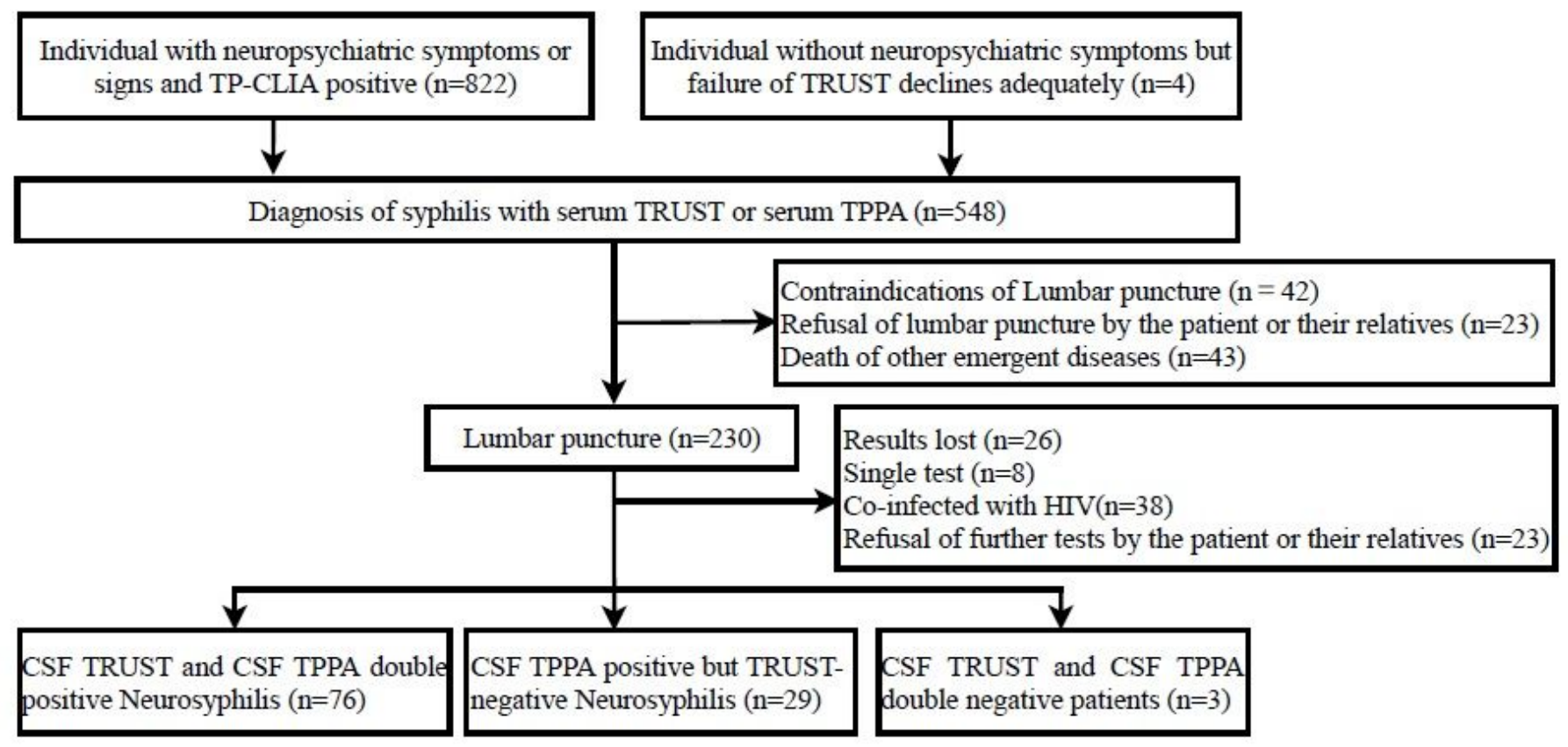

Figure 1

Participant flow diagram 


\section{Compare 2 models}

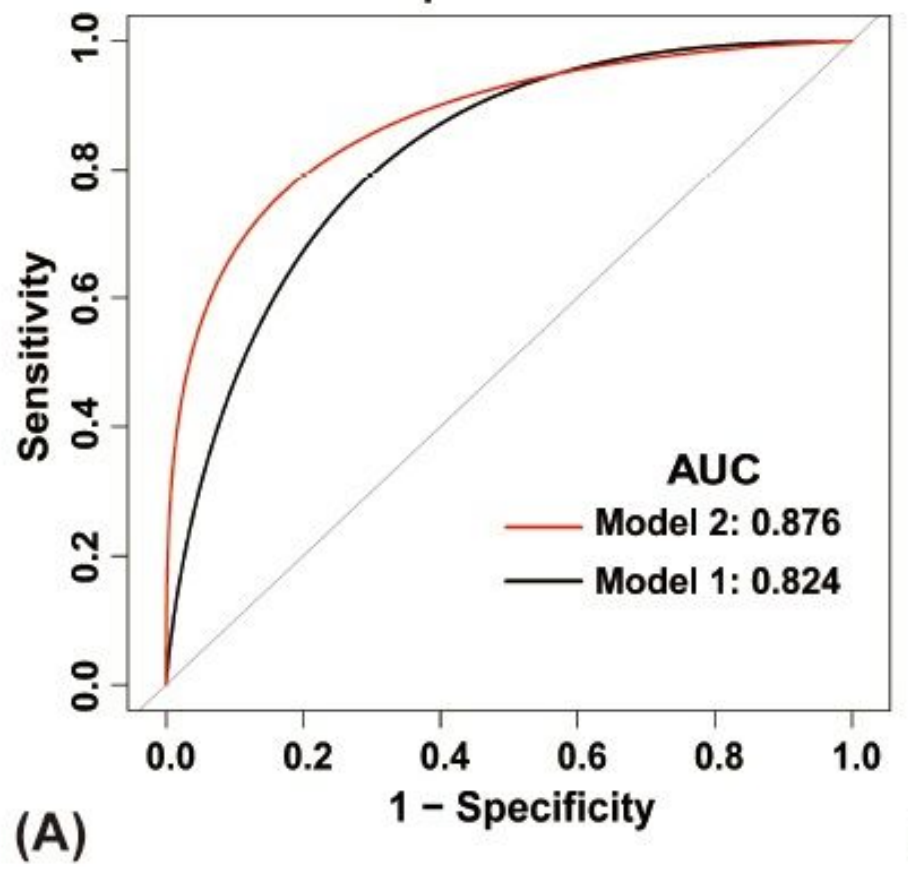

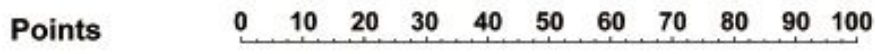

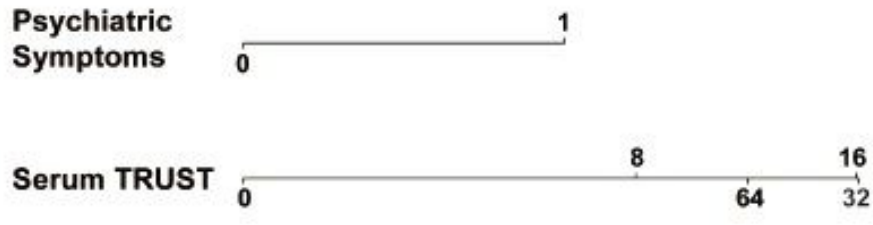

Total Points

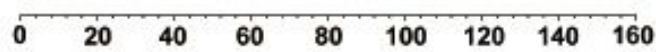

Linear

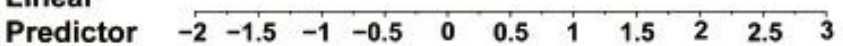

\section{Diagnostic Possibility}

of Neurosyphilis 0.2

(C)

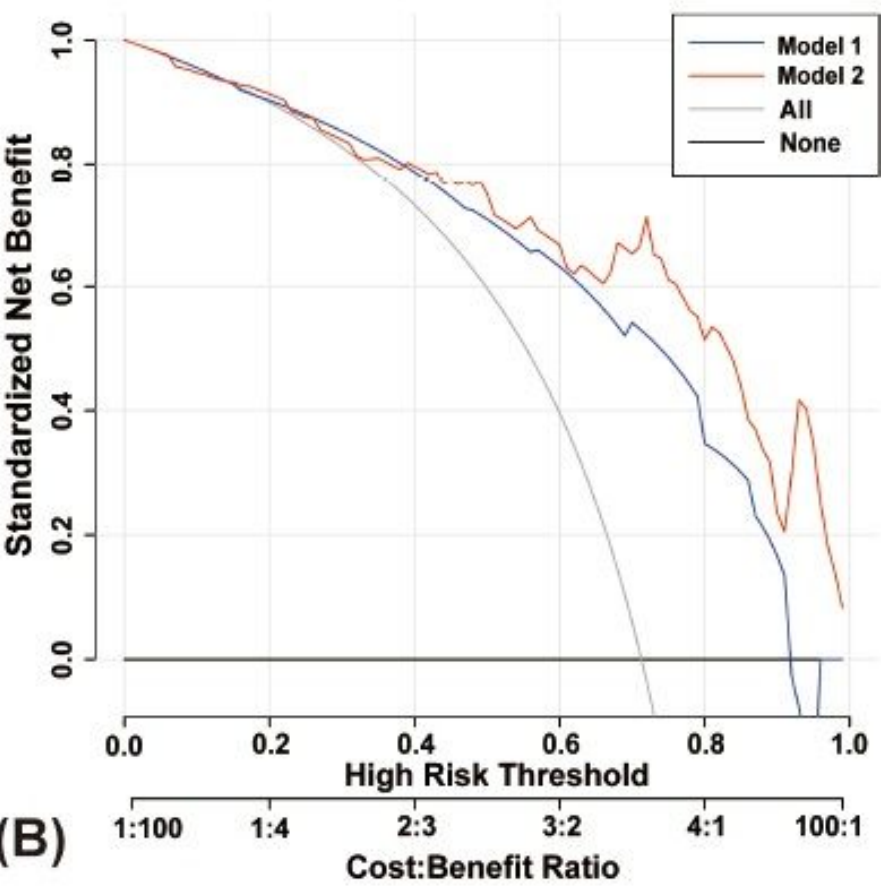

Points

$\begin{array}{lllllllllll}0 & 10 & 20 & 30 & 40 & 50 & 60 & 70 & 80 & 90 & 100\end{array}$ Psychiatric
Symptoms

Serum TRUST

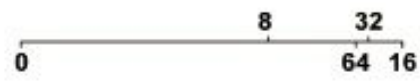

CSF Protein

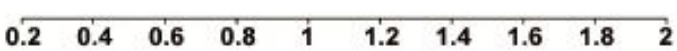

CSF Glucose

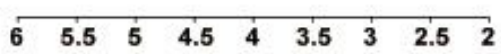

Total Points

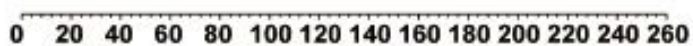

Linear Predictor $\begin{array}{llllllllllll}-4 & -3 & -2 & -1 & 0 & 1 & 2 & 3 & 4 & 5 & 6 & 7\end{array}$

Diagnostic Possibility of Neurosyphilis

$0.1 \quad 0.5+0.9$

(D)

Figure 2

ROC curve, DCA and nomograms. (A) AUC of Model 1 (Basic model) and Model 2 (Combined model). P value $=0.02$ in comparison between Model 1 and Model 2. (B) DCA of Model 1 and 2 (C) A nomogram visualizing the multi-variable diagnostic model of Model 1. (D) Nomogram of Model 2. *AUC confidence interval and significance test adopt Bootstrap resampling 500 times 